\title{
Numerical Simulation by High-Order Explicit Finite Difference Method to Solve the Burgers Equation
}

\author{
Letícia Helena Paulino de Assis, Estaner Claro Romão* \\ University of São Paulo, Engineering School of Lorena, Environmental and Basic Sciences Department, São \\ Paulo, Brazil.
}

* Corresponding author. Tel.: 55-12-3159-5118; email: estaner23@usp.br, leticia.hpa@alunos.eel.usp.br Manuscript submitted January 12, 2019; accepted April 11, 2019.

doi: 10.17706/ijapm.2019.9.3.135-143

\begin{abstract}
This paper aims to apply the High Order Explicit Finite Difference Method to solve the famous Nonlinear 1D Burgers Equation in many orders in time (first, second, third and fourth), changing the order on space twice (second and fourth). Thereby, it was compared the results and it was found the best refinement. Thus, it is expected that this work not only can present or even confirm that in most cases greater refinements imply better numerical precision, but rather serve as a basis for decision-making when analyzing the best spatial and should be considered for numerical accuracy and low computational time.
\end{abstract}

Key words: Burgers equation, numerical simulation, numerical efficiency, transient state, explicit finite difference.

\section{Introduction}

According to [1] in situations where the diffusive effects are small in relation to the convective, the values of the first derivative distributed approach the imaginary axis of the complex plane in time. A method of time integration is a region of stability included in the imaginary axis and then necessary. Even though an explicit method is a stability domain that includes part of the imaginary axis, there is the problem of a maximum allowed time step. This is indeed the case with all explicit methods. It is necessary to use implicit methods to achieve unconditional stability. The author also showed that in dealing with problems of pure convection, it is usually possible to express the second derivative of the unknown in terms of spatial derivatives.

In concluding the paper on the use of Padé approximations, he says that it is an exponential function provided with good results for both the implicit and the explicit high order method. In conclusion, it is confirmed that meshless methods are still being investigated with regard to their stability and precision in highly convective transport problems.

In [2] the mesh method is used to study the rapid cooling of strawberry with forced air. At the end of the experiment, it was observed that the lower result fit for the polynomial order. However, a "curve" that best represents the physical phenomenon of the process for an order-of-order adjustment, the adjustment being selected. A numerical simulations methodology was promising, since it presents as information as a basis for the development of equipment in the fast cooling area with forced air of spherical fruits, it presupposes a value of the conventional coefficient of heat transfer and determination of the time of strawberry cooling. It was noted that the stability condition should be observed when using the explicit method.

For [3] the explicit method can be trivially expanded for problems in the dimension of isoperimetric 
spaces, which is not as easy a problem when implicit methods are essential. The algorithm studied led to numerical solutions that are in excellent condition as exact solutions. The author concluded that he uses a Fourier-von Neumann technique, terms of conditions for which the fractional FTCS (stable fronted space) method is stable. For a conclusion, consider the time steps, in which the evaluation of the state of the system in a certain period of time requires information about all previous states and not just the previous affiliate, as occurs in the common diffusion, that is, there is a interdependence of the discrete temporal and spatial steps, one of the two for fixed.

According to [4] which compares the use of the spectral method with that of second-order final meshes, it was concluded that the methods of analysis are not suitable for the calculation of thin inner layers, make up the studied equation, Burgers with small viscosity, thin layer). If a sufficient number of modes are used and if a good transformation of departments or domain decomposition to projected, a precision in the critical region is quite acceptable. Numeric schemes, such as terminals, are also based on coordinate transformation, but suffer from numerical dispersion. The methods are better from the point of view and require higher order time schedules. However, as the reported calculations were performed on different computers, it is extremely difficult to relate the computing times of the various methods. Consequently, it is impossible to make a precise comparison between efficiency algorithms.

Besides that, according to [5] whose article presents a finite element schema of second order precision to simulate a Burgers equation, as direct comparisons between analytical and numerical solutions show that the proposed scheme has good precision. In addition, the asymptotic behaviour of indicative analysis solutions such as simulations maintains good accuracy over very large time intervals. The scenario used takes advantage as well convective and diffusive of this equation, use a complete equation and as initial conditions. It was concluded that good accuracy and a database on the proposed development of the device. The appropriate method to produce information about the analytical good of the solutions of the Burgers equation.

In the article by [6] it is suggested a modification of the explicit method of finite differences for the evaluation of derivative securities. The modification ensured that, as smaller time intervals are taken, the convergence of the calculated values to the correct solution. It has been proven that the explicit method is easier to implement and conceptually simpler. The disadvantage of the explicit method is that it does not necessarily converge. It has been found that the explicit method uses between 40 and 70 percent of the time the implicit time uses to provide the same level of accuracy. One of the reasons for the extra efficiency of the explicit method is that most of the problems studied in the article are initial value problems, not threshold value problems. Errors are introduced by redundant boundary conditions in implicit methods.

The article by [7] presents an analytical solution by the finite diffraction method based on the explicit standard method for the one-dimensional Burgers equation that frequently arises in the mathematical modelling used to solve problems in fluid dynamics. The results obtained by these modes for some modest viscosity values were compared with the exact (Fourier). In conclusion, since all the numerical results obtained by the methods show a reasonably good agreement with the exact modest viscosity values, and also exhibit the expected convergence as the mesh size is decreased, both methods can therefore be considered competitive and recommended. The current solution is an alternative solution for the exact (Fourier). But if the initial condition of a problem is known only in the finite number of mesh points, for this problem the current solution method is much more practical than Fourier.

According to [8] the fourth order finite difference method for the solution of the one-dimensional non-linear Burgers equation in space and second-order precision in time was effective for any Reynolds value. The method was presented simple and precise to solve the Burgers equation, although unconditionally stable. The convergence analysis of the method was studied and an upper bound for the 
error was derived. The solution found was an alternative solution for the exact (Fourier). But if the initial condition of a problem known only in the finite number of the mesh point, for this problem, the present method is much more practical than the Fourier method. It is believed to be useful for solving more general problems in fluid dynamics.

In [9] an explicit time domain integration algorithm was used for the equations associated with elastodynamic problems, using the Finite Element Method. Fourth order finite differences were used, since high precision has been developed in order to use them for transient problems, as a way to improve the quality of the results and especially a significant reduction in the numerical oscillations, which was observed when comparing with the difference central. Other advantages offered by the explicit difference were found, namely: low memory usage and no need to assemble and solve the global system of equations. In addition, the time integration techniques presented as a good alternative to the methods of nodal superposition in the analysis of transient problems, the ease of implementation, the reduced computational cost in relation to the implicit method and the non-assembly of the system of equations.

Several other works [10]-[16] have sought to solve nonlinear problems in an implicit or explicit way, but one of the main questions that still arises is the best composition between spatial and transient discretization for solving nonlinear problems.

In this paper objective to present a study of the variation the two spatial and four time discretization and to demonstrate which of these formulations to present the better precision in the solution of the 1D Burgers Equation.

\section{Model Equation}

Burgers' equation is a fundamental partial differential equation occurring in various areas of applied mathematics, such as fluid mechanics, nonlinear acoustics, gas dynamics, traffic flow,

$$
\frac{\partial u}{\partial t}+u \frac{\partial u}{\partial x}=v \frac{\partial^{2} u}{\partial x^{2}}
$$

where $v>0$ is the coefficient of kinematic viscosity.

\section{Numerical Formulation}

Before starting the numerical formulation of Equation (1), we rearrange these equations as follows,

$$
\frac{\partial u}{\partial t}=v \frac{\partial^{2} u}{\partial x^{2}}-u \frac{\partial u}{\partial x}
$$

where $v$ is the viscosity $\left(\mathrm{m}^{2} / \mathrm{s}\right)$.

\subsection{Spatial Discretization}

For Spatial Discretization will be to used two discretization forms: Second and Fourth Order Finite Difference Methods. To Second order will be to used the expressions,

$$
\begin{aligned}
& \frac{\partial u}{\partial x}=\frac{u_{i+1}-u_{i-1}}{2 \Delta x} \\
& \frac{\partial^{2} u}{\partial x^{2}}=\frac{u_{i+1}-2 u_{i}+u_{i-1}}{\Delta x^{2}}
\end{aligned}
$$

that substitute in Equation (2) results in 


$$
\frac{\partial u_{i}^{n+1}}{\partial t}=v\left(\frac{u_{i+1}^{n}-2 u_{i}^{n}+u_{i-1}^{n}}{\Delta x^{2}}\right)-u_{i}^{n}\left(\frac{u_{i+1}^{n}-u_{i-1}^{n}}{2 \Delta x}\right)
$$

where " $n+1 "$ is current time step and " $n$ " is the previous. Now to the case where is used Fourth Order precision, follow the expressions,

$$
\begin{aligned}
& \frac{\partial u}{\partial x}=\frac{-u_{i+2}+8 u_{i+1}-8 u_{i-1}+u_{i-2}}{12 \Delta x} \\
& \frac{\partial^{2} u}{\partial x^{2}}=\frac{-u_{i+2}+16 u_{i+1}-30 u_{i}+16 u_{i-1}-u_{i-2}}{12 \Delta x^{2}}
\end{aligned}
$$

that substitute in Equation (2) results in

$$
\frac{\partial u_{i}^{n+1}}{\partial t}=v\left(\frac{-u_{i+2}^{n}+16 u_{i+1}^{n}-30 u_{i}^{n}+16 u_{i-1}^{n}-u_{i-2}^{n}}{\Delta x^{2}}\right)-u_{i}^{n}\left(\frac{-u_{i+2}^{n}+8 u_{i+1}^{n}-8 u_{i-1}^{n}+u_{i-2}^{n}}{2 \Delta x}\right)
$$

\subsection{Temporal Discretization}

In this paper, the Finite Difference Method is used as an approximation of the temporal partial derivatives in the Burgers equation with finite differences that relate the values of the unknown function at a set of neighbouring grid points at various time levels. This approximation replaces the partial differential equations with a finite difference equation with

$$
\frac{\partial u}{\partial t}=\lim _{\Delta t \rightarrow 0} \frac{u(t+\Delta t)}{\Delta t}
$$

That if in Equation (9) $u(t+\Delta t)$ is expanded in Taylor series around $t$, results in

$$
u(t+\Delta t)=u(t)+\Delta t \frac{\partial u(t)}{\partial t}+\frac{(\Delta t)^{2}}{2 !} \frac{\partial^{2} u(t)}{\partial t^{2}}+\frac{(\Delta t)^{3}}{3 !} \frac{\partial^{3} u(t)}{\partial t^{3}}+\cdots
$$

Substituting the Equation (10) in the Equation (9), obtains

$$
\frac{u(t+\Delta t)-u(t)}{\Delta t}=\frac{\partial u(t)}{\partial t}+\frac{\Delta t}{2 !} \frac{\partial^{2} u(t)}{\partial t^{2}}+\frac{(\Delta t)^{2}}{3 !} \frac{\partial^{3} u(t)}{\partial t^{3}}+\cdots=\frac{\partial u(t)}{\partial t}+O(\Delta t)
$$

where $O(\Delta t)$ is the first order truncation error.

For discretization of the left term in Equation (5) or (8) will be used four schemes: First, Second, Third and Fourth Backward Finite Difference. To the three first approximations, follow the expressions conform [17]:

$$
\begin{gathered}
\frac{\partial u_{i}^{n+1}}{\partial t}=\frac{u_{i}^{n+1}-u_{i}^{n}}{\Delta t} \\
\frac{\partial u_{i}^{n+1}}{\partial t}=\frac{3 u_{i}^{n+1}-4 u_{i}^{n}+u_{i}^{n-1}}{2 \Delta t} \\
\frac{\partial u_{i}^{n+1}}{\partial t}=\frac{11 u_{i}^{n+1}-18 u_{i}^{n}+9 u_{i}^{n-1}-2 u_{i}^{n-2}}{6 \Delta t}
\end{gathered}
$$


First, second and third Backward Finite Difference, respectively.

Now, for fourth order approximations will be utilized the follow expressions, that are written from Taylor series of the follow forms,

$$
\begin{gathered}
u^{n}=u^{n+1}-\Delta t \frac{\partial u(t)}{\partial t}+\frac{(\Delta t)^{2}}{2 !} \frac{\partial^{2} u(t)}{\partial t^{2}}-\frac{(\Delta t)^{3}}{3 !} \frac{\partial^{3} u(t)}{\partial t^{3}}+\cdots \\
u^{n-1}=u^{n+1}-2 \Delta t \frac{\partial u(t)}{\partial t}+\frac{4(\Delta t)^{2}}{2 !} \frac{\partial^{2} u(t)}{\partial t^{2}}-\frac{8(\Delta t)^{3}}{3 !} \frac{\partial^{3} u(t)}{\partial t^{3}}+\cdots \\
u^{n-2}=u^{n+1}-3 \Delta t \frac{\partial u(t)}{\partial t}+\frac{9(\Delta t)^{2}}{2 !} \frac{\partial^{2} u(t)}{\partial t^{2}}-\frac{27(\Delta t)^{3}}{3 !} \frac{\partial^{3} u(t)}{\partial t^{3}}+\cdots \\
u^{n-3}=u^{n+1}-4 \Delta t \frac{\partial u(t)}{\partial t}+\frac{16(\Delta t)^{2}}{2 !} \frac{\partial^{2} u(t)}{\partial t^{2}}-\frac{64(\Delta t)^{3}}{3 !} \frac{\partial^{3} u(t)}{\partial t^{3}}+\cdots
\end{gathered}
$$

that when used together can construct the following expression

$$
\begin{gathered}
a u^{n+1}+b u^{n}+c u^{n-1}+d u^{n-2}+e u^{n-3}=(a+b+c+d+e) u^{n+1}-\Delta t(b+2 c+3 d+4 e) \frac{\partial u(t)}{\partial t}+ \\
\frac{(\Delta t)^{2}}{2 !}(b+4 c+9 d+16 e) \frac{\partial^{2} u(t)}{\partial t^{2}}-\frac{(\Delta t)^{3}}{3 !}(b+8 c+27 d+64 e) \frac{\partial^{3} u(t)}{\partial t^{3}}+\frac{(\Delta t)^{4}}{4 !}(b+16 c+81 d+256 e) \frac{\partial^{4} u(t)}{\partial t^{4}}
\end{gathered}
$$

To find the first temporal derivative of $u$ with Fourth Order accuracy, we find the following system (assuming null the coefficients of terms of derivatives of higher order):

$$
\begin{gathered}
a+b+c+d+e=0 \\
b+2 c+3 d+4 e=-1 \\
b+4 c+9 d+16 e=0 \\
b+8 c+27 d+64 e=0 \\
b+16 c+81 d+256 e=0
\end{gathered}
$$

where the solution of linear system is $a=25 / 12, b=-4, c=3, d=-4 / 3$ and $e=1 / 4$. From this solution we have the expression for the finite difference backward of $O\left(\Delta t^{4}\right)$ will be:

$$
\frac{\partial u}{\partial t}=\frac{25 u^{n+1}-48 u^{n}+36 u^{n-1}-16 u^{n-2}+3 u^{n-3}}{12 \Delta t}
$$

\section{Numerical Applications}

FORTRAN codes were made using the Equations (5), (8), (12), (13), (14) and (20). First, it was made four different codes on spatial second order with the four different orders in time in each code. Then, it was made the same for fourth order in space. 
Application 1: For this application, supposing $v=1$, it was used the following exact solution:

$$
u(x, t)=\frac{2 x}{1+2 t}
$$

To analyze the results, will be used the $L_{\infty}$ norm from the comparison with the exact solution. In this application will be used $0 \leq x \leq 1$ and $0 \leq t \leq 1$. In this application (Table 1 ) is demonstrate that the numerical results present a relation with the term $\Delta t / \Delta x^{2}$, in special, the greater this term, more fast the variation of $\Delta x$ leads to divergent results. Note that the convergent occurs only for $\Delta t / \Delta x^{2}$ smaller than 0.5 and the higher the order of accuracy in time smaller will be the value of $\Delta t / \Delta x^{2}$ for convergence.

Table 1. $L$ Norm Varying the Discretization in Time and Space

\begin{tabular}{cccccccccc}
\hline \hline \multicolumn{9}{c}{$O(\Delta x)^{2}$} & \multicolumn{3}{c}{$O(\Delta x)^{4}$} & \multicolumn{1}{c}{${ }^{4}$} \\
$\Delta x$ & $O(\Delta t)$ & $O(\Delta t)^{2}$ & $O(\Delta t)^{3}$ & $O(\Delta t)^{4}$ & $O(\Delta t)$ & $O(\Delta t)^{2}$ & $O(\Delta t)^{3}$ & $O(\Delta t)^{4}$ & $\Delta x^{2}$ \\
\hline $\mathbf{0 . 1}$ & $2.288 \mathrm{E}-06$ & $9.148 \mathrm{E}-06$ & $1.372 \mathrm{E}-05$ & $1.829 \mathrm{E}-05$ & $2.285 \mathrm{E}-06$ & $9.141 \mathrm{E}-06$ & $1.370 \mathrm{E}-05$ & $1.827 \mathrm{E}-05$ & 0.01 \\
$\mathbf{0 . 0 5}$ & $2.286 \mathrm{E}-06$ & $9.142 \mathrm{E}-06$ & $1.371 \mathrm{E}-05$ & $1.827 \mathrm{E}-05$ & $2.285 \mathrm{E}-06$ & $9.140 \mathrm{E}-06$ & $1.370 \mathrm{E}-05$ & $1.827 \mathrm{E}-05$ & 0.04 \\
$\mathbf{0 . 0 3 3 3}$ & $2.287 \mathrm{E}-06$ & $9.148 \mathrm{E}-06$ & $1.371 \mathrm{E}-05$ & $1.828 \mathrm{E}-05$ & $2.287 \mathrm{E}-06$ & $9.147 \mathrm{E}-06$ & $1.371 \mathrm{E}-05$ & DIV & 0.09 \\
$\mathbf{0 . 0 2 5}$ & $2.289 \mathrm{E}-06$ & $9.154 \mathrm{E}-06$ & DIV & DIV & $2.288 \mathrm{E}-06$ & $9.153 \mathrm{E}-06$ & DIV & DIV & 0.16 \\
$\mathbf{0 . 0 2}$ & $2.289 \mathrm{E}-06$ & $9.155 \mathrm{E}-06$ & DIV & DIV & $2.289 \mathrm{E}-06$ & DIV & DIV & DIV & 0.25 \\
$\mathbf{0 . 0 1 6 6 6 7}$ & $2.289 \mathrm{E}-06$ & DIV & DIV & DIV & $2.289 \mathrm{E}-06$ & DIV & DIV & DIV & 0.36 \\
$\mathbf{0 . 0 1 4 2 8 6}$ & $2.289 \mathrm{E}-06$ & DIV & DIV & DIV & DIV & DIV & DIV & DIV & 0.49 \\
$\mathbf{0 . 0 1 2 5}$ & DIV & DIV & DIV & DIV & DIV & DIV & DIV & DIV & 0.64 \\
$\mathbf{0 . 0 1 1 1}$ & DIV & DIV & DIV & DIV & DIV & DIV & DIV & DIV & 0.81 \\
$\mathbf{0 . 0 1}$ & DIV & DIV & DIV & DIV & DIV & DIV & DIV & DIV & 1 \\
\hline \hline
\end{tabular}

DIV: divergent

Application 2: In this application it was considered $v=1$ with the following boundary and initial condition: $u(0, t)=u(1, t)=0$ and $u(x, 0)=\sin (\pi x)$. The results of $u(x, t)$ by the simulation were compared to exact results. The best approximation was for the spatial fourth order and temporal fourth order, followed by spatial fourth order and temporal third order (Table 2 and 3). The results that reached very close to the exact solution were time more refined, due to the condition of explicit method that only works in a specific range of values.

Table 2. Values of $u(x, t)$ for $\Delta t=10^{-5}$ and $\Delta x=10^{-1}$ in Temporal and Spatial Fourth Order

\begin{tabular}{ccc}
\hline \hline $\boldsymbol{x}$ & Present work & Exact [18] \\
\hline 0.1 & 0.10923 & 0.10953 \\
0.2 & 0.20916 & 0.20979 \\
0.3 & 0.29100 & 0.29189 \\
0.4 & 0.34687 & 0.34792 \\
0.5 & 0.37047 & 0.37157 \\
0.6 & 0.35802 & 0.35904 \\
0.7 & 0.30907 & 0.3099 \\
0.8 & 0.22727 & 0.22781 \\
0.9 & 0.12048 & 0.12068 \\
\hline \hline
\end{tabular}

Table 3. Values of $u(x, t)$ for $\Delta t=2 \times 10^{-5}$ and $\Delta x=10^{-1}$ in Temporal Third Order and Spatial Fourth Order

\begin{tabular}{ccc}
\hline \hline $\boldsymbol{x}$ & Present work & Exact [18] \\
\hline 0.1 & 0.10955 & 0.10953 \\
0.2 & 0.20978 & 0.20979 \\
0.3 & 0.29186 & 0.29189 \\
0.4 & 0.34788 & 0.34792 \\
\hline \hline
\end{tabular}




\begin{tabular}{lll}
\hline \hline 0.5 & 0.37155 & 0.37157 \\
0.6 & 0.35905 & 0.35904 \\
0.7 & 0.30996 & 0.30990 \\
0.8 & 0.22792 & 0.22781 \\
0.9 & 0.12082 & 0.12068 \\
\hline \hline
\end{tabular}

Despite of the high refinement in time, the spatial one was not expressive. This mash in these conditions was efficient since the results got very close from the exact solution.

Application 3: In this application, as the previous one, it was considered $v=1$ with the following boundary and initial condition: $u(0, t)=u(1, t)=0$ and $u(x, 0)=4 x(1-x)$. The results of $u(x, t)$ by the simulation were compared to exact results. The best approximation was for the spatial and time fourth order. The results are presented in Table 4.

Table 4. Values of $u(x, t)$ for $\Delta t=2 \times 10^{-6}$ and $\Delta x=2.5 \times 10^{-3}$ in Temporal and Spatial Fourth Order

\begin{tabular}{cccc}
\hline \hline $\boldsymbol{x}$ & $\boldsymbol{t}$ & Present work & Exact [19] \\
\hline \multirow{3}{*}{0.25} & 0.10 & 0.26146 & 0.26148 \\
& 0.15 & 0.16146 & 0.16148 \\
& 0.20 & 0.09946 & 0.09947 \\
& 0.25 & 0.06108 & 0.06108 \\
0.50 & 0.10 & 0.38340 & 0.38342 \\
& 0.15 & 0.23403 & 0.23406 \\
& 0.20 & 0.14287 & 0.14289 \\
& 0.25 & 0.08722 & 0.08723 \\
0.75 & 0.10 & 0.28156 & 0.28157 \\
& 0.15 & 0.16972 & 0.16974 \\
& 0.20 & 0.10264 & 0.10266 \\
& 0.25 & 0.06228 & 0.06229 \\
\hline \hline
\end{tabular}

The results present in Table 4 demonstrate that this formulation have a excellent precision, achieving order precision of $10^{-5}$.

\section{Conclusion}

In this paper, it was observed that the best discretization in time and in space depends on the application due to the imprecision of explicit method that make its works only in a specific range of values. In general, the best results become from the high order discretization, as we saw in three applications in this work. It was concluded that the method is efficient and attend the resolution of systems, since the results were almost the same of the exact solutions.

\section{Acknowledgment}

The FAPESP (Procs. 2014/06679-8 and 2016/16867-1) and CNPq (Proc. 400898/2016-0) supported the present work.

\section{References}

[1] Donea, J., Roig, B., \& Huerta, A. (2000). High-order accurate time-stepping schemes for convection-diffusion problems. Computer Methods in Applied Mechanics and Engineering, 182(3-4), 249-275.

[2] Pirozzi, D. C. Z., \& Amendola, M. (2005). Modelagem matemática e simulação numérica do resfriamento rápido de morando com ar forçado. Engenharia Agrícola, Jaboticabal, 25(1), 222-230.

[3] Yuste, S. B., \& Acedo, L. (2005). An explicit finite difference method and a new von neumann-type stability analysis for fractional diffusion equations. Elsevier Science, 42(05), 1-22.

[4] Basdevant, C., Deville, M. O., \& Haldenwang, P. (1986). Spectral and finite difference solutions of the 
burgers equation. Computers \& Fluids, 14(1), 23-41.

[5] Konzen, P. H. A., Sauter, E., Azevedo, F., \& Zingano, P. R. A. (2016). Numerical simulations with the finite element method for the Burgers' equation on the real line. Tendências em Matemática Aplicada e Computacional, 18(2), 287-304.

[6] Hull, J., \& White, A. (1990). Valuing derivative securities using the explicit finite difference method. The Journal of Financial and Quantitative Analysis, 25(1), 87-100.

[7] Kutluay, S., Bahadir, A. R., \& Özdes, A. (1999). Numerical solution of one-dimensional Burgers equation: Explicit and exact-explicit finite difference methods. Journal of Computational and Applied Mathematics, 103(2), 251-261.

[8] Hassanien, I. A., Salama, A. A., \& Hosham, H. A. (2005). Fourth-order finite difference method for solving Burgers' equation. Applied Mathematics and Computation, 170(2), 781-800.

[9] Souza, L. A., \& Moura, C. A. (1997). Diferença finita de quarta ordem para integração explicita no domínio do tempo de problemas elastodinâmicos. XVIII CILAMCE, Brasília -DF, 1. 263-272.

[10] Neves, O. A., Romão, E. C., Silva, J. B. C., \& Moura, L. F. M. (2011). Numeric simulation of pollutant dispersion by a control-volume based on finite element method. International Journal for Numerical Methods in Fluids, 66, 1073-1092.

[11] Srivastava, V. K., Tamsir, M., Bhardwaj, U., \& Sanyasiraju, Y. V. S. S. (2011). Crank-Nicolson scheme for numerical solutions of two-dimensional coupled burgers' equations. Int. J. Sci. \& Eng. Research, 2(5), 1-7.

[12] Campos, M. D., \& Romão, E. C. (2014). A high-order finite-difference scheme with a linearization technique for solving of three-dimensional burgers equation. Computer Modeling in Engineering \& Sciences, 103, 139-154.

[13] Bahadir, A. R. (2003). A fully implicit finite-difference scheme for two dimensional Burgers equations. Appl. Math. Comput., 137, 131-137.

[14] Neves, O. A., Romão, E. C., Silva, J. B. C., \& Moura, L. F. M. (2010). Numerical simulation of convection-diffusion problems by the control-volume based finite-element method. Numerical Heat Transfer. Part A, Applications, 57, 730-748.

[15] Radwan, S. F. (2005). Comparison of higher-order accurate schemes for solving the two-dimensional unsteady Burgers' equation. J. Comput. Appl. Math., 174, 383-397.

[16] Romão, E. C., \& Moura, L. F. M. (2013). 3D contaminant transport by GFEM with hexahedral elements. International Communications in Heat and Mass Transfer, 42, 43-50.

[17] Chung, T. J. (2002). Computational Fluid Dynamics. Cambridge: Cambridge University Press.

[18] Xu, M., Wang, R. H., Zhang J., \& Fang Q. (2011). A novel numerical scheme for solving Burgers' equation. Appl. Math. Comput., 217, 4473-4482.

[19] Cruz, M. M., Campos, M. D., Martins, J. A., \& Romão, E. C. (2014). An efficient technique of linearization towards fourth order finite differences for numerical solution of the 1D burgers equation. Defect and Diffusion Forum, 348, 285-290.

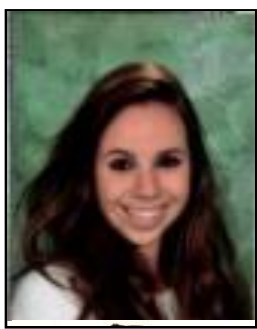

Letícia Helena Paulino de Assis is a student of the chemical engineering of the Engineering School at Lorena of University of São Paulo in Brazil. 


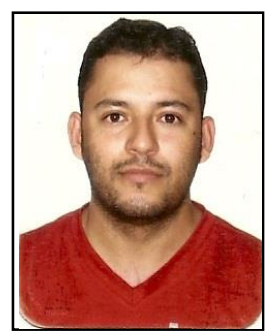

Estaner Claro Romão is professor doctor in Basic and Environmental Science Department of the Engineering School at Lorena of University of São Paulo in Brazil. Currently his research is on themes numerical simulation in heat and mass transfer problems. 